\title{
Medio ambiente, economía de subsistencia y patrones de asentamiento del Complejo Chiu Chiu (ca. 3000 a 2000 AC), norte de Chile ${ }^{1}$
}

Entre marzo de 1970 y agosto de 1971, el autor condujo una investigación de campo en la región Calama-Chiu Chiu en el Desierto de Atacama, norte de Chile, asesorado por National Science Foundation. ${ }^{3}$ El trabajo se efectuó en conexión con el Proyecto de los Andes de la Universidad de Columbia, con el Prof. E. P. Lanning, como director. El trabajo se concentró en la relación entre cambios ambientales en pequeña escala, economía de subsistencia y patrones de asentamiento del Período Precerámico Tardío del Complejo Chiu Chiu. La información reunida hasta ahora sugiere que el área osciló entre condiciones de extremo desierto y condiciones semiáridas durante el Período Chiu Chiu. Durante períodos secos como el presente, la principal zona de habitación estaba ubicada en las vegas del río Loa y era suficiente para mantener sólo unas pocas familias de cazadores-recolectores por períodos muy cortos cada año, cuando más. Durante los años más húmedos, aumentaba grandemente la capacidad de recursos de toda la región. La vegetación llegaba a áreas más bajas, la vega del Loa y los cerros de Tuina, área de recursos normalmente separadas por más de $20 \mathrm{~km}$ de extremo desierto. Ante estas condiciones ambas áreas se juntaban reduciendo el número y espacio de microambientes explotados por la población de Chiu Chiu, resultando una mayor permanencia de los asentamientos en las vegas principales del río Loa.

La información reunida antes de la temporada de campo 1970-1971 puede resumirse como sigue: el Complejo Chiu Chiu estaba representado por 70 sitios

1 Trabajo presentado en la $37^{\mathrm{a}}$ Reunión Anual de la Sociedad de Arqueología Americana, 4 a 6 de mayo de 1972, Miami. Traducción de Cora Moragas.

2 Ph. D. (C) Universidad de Columbia, Nueva York, ESTADOS UNIDOS.

3 Investigación asesorada por National Science Foundation Doctoral Dissertation Improvement Grant GS-2888, Chile Exploration Company, y la Compañía de Cobre de Chuquicamata S.A., bajo autorizaciones del Museo de Arqueología de San Pedro de Atacama, su director Gustavo Le Paige, la Universidad del Norte y el Consejo de Monumentos Nacionales. y subsitios centrados especialmente alrededor de los márgenes de dos lagos, ahora secos, desaguados por el río Loa. Las consideraciones tipológicas y cinco fechas radiocarbónicas situadas entre 2705 y 2060 AC ubican claramente este Complejo en la parte media del Precerámico de Lanning, en el Período V y la fase temprana del Período VI (Lanning y Hammel 1961; Lanning 1967).

El Complejo se caracteriza por puntas foliáceas grandes y pequeñas, doblepuntas y puntas lanceoladas. Las comparaciones preliminares entre los sitios revelan similitudes con las culturas del Anzuelo de Concha y del Segundo Período Precerámico descritos por Bird (1943) para la costa norte de Chile, Lauricocha III, el abrigo rocoso IV en Laguna Hedionda (Barfield 1961) y Viscachani (Patterson, com. pers.).

Entre otros artefactos se incluyen varios tipos de perforadores pequeños y microlíticos, algunos muy bien tallados a presión, matrices de perforadores, cuentas líticas, de hueso y de dientes, cordelería de lana de camélido, un palo cavador, piedras de molienda, morteros y manos, retocadores y cuchillos grandes muy bien trabajados. La mayoría de los artefactos líticos están hechos en calcedonia blanca de la localidad, o en rocas volcánicas. El basalto, que abunda a $30 \mathrm{~km}$ del río Loa, se usó para elaborar una minoría significativa de artefactos.

Los tipos de asentamientos incluyen sitios de canteras, talleres y campamentos, variando el tamaño de dos a más de $200 \mathrm{~m}$ de diámetro. Algunos de los campamentos más pequeños pudieron ser abrigos donde pernoctaban los cazadores, mientras los sitios mayores y más profundos pudieron haber sido ocupados en varias estaciones sucesivas o durante todo el año, o por varios años consecutivos, por unas cinco a ocho familias. En algunos sitios se encontraron restos de estructuras simples como plataformas de casas, círculos habitacionales o bases de piedra para resguardarse del viento, mientras que otros sitios poseían depresiones más hondas, que sugieren la posibilidad de casas semisubterráneas. 
Los restos de plantas recuperadas antes de la temporada de 1970 incluyen semillas de herbáceas y rizomas de juncos. También se encontraron huesos de cérvidos, camélidos, roedores, pájaros y pescados.

Una división preliminar por fases dentro del Complejo permitió incluir sitios grandes y pequeños, potentes y bajos, a través de la secuencia, sugiriendo la localización de actividades o cambios en el grado de sedentarismo o ambas posibilidades combinadas con un patrón de trashumancia estacional. Los vacíos en la seriación, entre el Complejo Chiu Chiu y los complejos más tempranos y más tardíos, sugieren la posibilidad de largos períodos de abandono de las vegas del Loa.

En la Puna, en Ojos de San Pedro, se encuentran evidencias de abandono estacional. Es un área de lagos, ríos y vertientes, ubicada aproximadamente a 48 millas de las vegas del Loa, con una altitud de 12480 pies. Hasta su reciente desagüe artificial, el área era habitada por centenares de patos, flamencos y otras aves acuáticas, además de vicuñas, vizcachas y zorros. La llareta, utilizada como combustible, estaba disponible hasta hace unos 30 a 40 años en las colinas bajas, en los alrededores de los volcanes, muy cercanos al área de Ojos del Salado. Un breve survey en la zona, en 1968, dio como resultado materiales muy parecidos a los de Chiu Chiu, sugiriendo que: a) Esta área estaba incluida en un patrón de trashumancia estacional, siendo la zona del Loa la parte más baja de término del ciclo; b) Esta área pudo servir de refugio durante los períodos en que las vegas del Loa eran improductivas.

La variabilidad de la productividad en las vegas del Loa estaría de acuerdo a cambios climáticos. Antes de la temporada de 1970-1971, varios miembros del Proyecto de los Andes habían colectado suficiente información para sugerir que había ocurrido un cambio climático local durante el Período Chiu Chiu. Nosotros también podemos predecir un aumento de humedad en supuestas tierras de suelos. El profesor Sarma (1969) ha relacionado el aumento de lluvias en la península de Santa Elena, Ecuador, a un patrón panandino frío pluvial. El aumento de humedad en la península de Santa Elena está correlacionado con el aumento de lluvias en las tierras altas andinas, a la vez que aumenta el afloramiento de aguas subterráneas en los sectores precordilleranos y costeros de Sudamérica occidental (Sarma 1969: 124, 131). Un período pluvial con tres máximos pluviales se infirió para Santa Elena entre 2650 y 1600 AC
(Sarma 1969: 116), y esto es contemporáneo por lo menos con parte de la secuencia de Chiu Chiu. Por eso, nosotros esperaríamos que las condiciones locales, no obstante, en la región de Calama-Chiu Chiu, en la precordillera andina meridional, habrían sufrido similares oscilaciones secas y húmedas durante por lo menos parte de la ocupación en Chiu Chiu. Hipotéticamente estas oscilaciones habrían causado cambios en la densidad de vegetación, tiempos de germinación y localización de mayor altura de vegetación y nieves en la precordillera y tierras altas, afectando así la capacidad de las áreas productivas del Complejo Chiu Chiu y el grado de permanencia y de duración del asentamiento Chiu Chiu en la zona principal.

La temporada de campo 1970-1971 se realizó para probar esta hipótesis. Se condujeron excavaciones en cinco sitios para determinar el grado de permanencia y en qué estación fueron ocupados, para complementar la información ya existente sobre subsistencia y para extraer muestras líticas y radiocarbónicas estratificadas y evidencia de cambios climáticos, que estarían unidos estratigráficamente a la ocupación de Chiu Chiu. Se tomó mucho tiempo en investigar los sitios de los alrededores de la región para localizar las áreas de recursos explotados. Este proyecto abarcó discusiones con mineros y otros residentes antiguos en la localidad, acerca del ganado de otros tiempos y las condiciones climáticas. De acuerdo a un permiso especial, cazamos guanacos, vizcachas, cholulos o tucu-tucu (un roedor similar al topo pequeño), patos y taguas gigantes (un ave acuática de gran tamaño) para poder observar hábitos en los animales y obtener muestras identificadas de esqueletos, plumas y pieles con propósitos comparativos. ${ }^{4}$ También se colectaron muestras de flora silvestre local. Se designaron prospecciones de sitios adicionales en regiones cercanas para verificar la hipótesis de campo acerca de la trashumancia estacional.

Excavamos un total de 22 unidades, la mayoría de $2 \times 2 \mathrm{~m}$, variando en profundidad de $20 \mathrm{~cm}$ a $2 \mathrm{~m}$. Se recuperaron más de 4000 artefactos diagnósticos, 73 muestras radiocarbónicas asociadas a material cultural, 20 muestras de suelos y algunos miles de restos de plantas y huesos de animales, que complementaron los datos excavados en temporadas previas.

4 Autorización concedida bajo la sección 8 de la ley chilena de caza y pesca. 
Como se esperaba, el grado de permanencia de los asentamientos varió considerablemente. Se descubrió un abrigo para pernoctar en el sitio RAnL-140, mientras que se vio la posibilidad de ocupación estacional en el sitio 217-c, por las capas alternadas de 2 a $3 \mathrm{~cm}$ de material cultural compacto entre capas de arena eólica y plantas. Existe evidencia clara de ocupación intermitente en Confluencia 1 (RAnL-92), donde las excavaciones de la Universidad de Chile (Santiago) han dejado al descubierto tres estratos culturales claramente separados por capas de arena eólica estéril. Las ocupaciones de larga duración se sugieren por la estratigrafía del sector principal de RAnL-140, donde el depósito fértil es de $1.7 \mathrm{~m}$ de profundidad y no está visiblemente alterado por arena eólica. La ocupación aquí pudo ser estacional por algunos años (Lanning, com. pers.), o quizás anual (Davis, com. pers.). ${ }^{5}$

En las excavaciones esperábamos encontrar indicaciones definidas sobre ocupación anual, casas semisubterráncas bien delineadas, en los sitios 217 , 289 y 276 , pero no fue así. Los restos de estructuras en estos sitios eran mínimos. Depresiones naturales habían sido levemente modificadas, agregándole unas pocas piedras y ramas, y no eran muy profundas. De este modo, no tenemos evidencias arquitectónicas de un sedentarismo bien establecido.

Los datos sobre subsistencia incluyen otras especies previamente no registradas o no identificadas, como Trochocopus macultus (pejeperro), un pez de la costa, vizcacha de montaña y tucu-tucu, así como fauna ya recuperada en excavaciones anteriores. ${ }^{6}$ No se identificaron huesos de peces de río.

Los restos de plantas identificadas a la fecha consisten sólo en especies industriales y semillas de hierbas. A nuestro pesar, el algarrobo y el chañar están totalmente ausentes en las basuras. Supuestamente estas plantas crecieron en el área durante los tiempos de Chiu Chiu y no debieron ser ignoradas si es que

5 El autor agradece a la Dra. Emma Lou Davis por asistir una semana a la temporada de campo de la Universidad de Columbia-Universidad del Norte. Durante ese tiempo visitamos sitios arqueológicos y varios tipos de zonas microambientales para evaluar la potencialidad de los recursos de la región bajo condiciones actuales. Este reconocimiento ayudó al autor a formular hipótesis de campo sobre la trashumancia estacional del Complejo Chiu Chiu.

6 El autor agradece al Dr. Pequeño, de la Sección de Biología del Museo Nacional de Historia Natural (Santiago) por su identificación y por permitir fotografiar el espécimen tipo. estaban disponibles. Actualmente éstas maduran entre marzo y abril y su ausencia es tentativamente tomada como una evidencia de abandono de los sitios durante estos meses, sugiriendo así trashumancia estacional.

Se logró información adicional sobre trashumancia estacional especialmente como resultado de conversaciones con la Dra. Davis. Después de visitar varias zonas microambientales a lo largo de la costa y tierras altas junto al autor, la Dra. Davis sugirió la posibilidad que la trashumancia de Chiu Chiu fuera en tierras altas. Esto se puede confirmar en los Ojos de San Pedro, donde la Dra. Davis localizó un componente Chiu Chiu, mientras el autor excavaba un esqueleto de camélido. El autor volvió al área varios meses después con un pequeño grupo y ubicó 16 sitios líticos durante una semana de prospecciones en localidades cercanas a lagos, al río. Son posibles canteras y abrigos, en un área de $30 \mathrm{~km}^{2}$. El único componente Chiu Chiu ubicado pertenece a una de las fases representadas en el sitio 92 que sugiere que mientras la capa estéril de arena estaba siendo depositada en la confluencia del Loa-Salado (elevación de 7500 pies), la población estaba en tierras altas, en los Ojos de San Pedro (elevación de 12480 pies), posiblemente cazando vicuñas, patos y taguas, recolectando huevos de flamencos $\mathrm{y}$ atrapando vizcachas.

El material de Chiu Chiu también se encontró a 60 km al sureste del río Loa, cerca de Hierba Buena. Se encontraron lascas de calcedonia blanca asociadas a refugios contra el viento en los cerros de Tuina, a $40 \mathrm{~km}$ al sureste de su fuente probable de recursos: el área del río Loa.

La trashumancia estacional y otros patrones de asentamiento humano no pueden ser totalmente comprendidos sin referirse a las áreas de explotación, y fue en la localización e investigación de las áreas de recursos del Complejo Chiu Chiu donde la temporada de campo de 1970-1971 tuvo más éxito. Las prospecciones aéreas y terrestres ${ }^{7}$ junto a nuestro proyecto de caza y recolección, nos permitieron reconocer a los cerros de Tuina como una de las áreas principales de recursos locales para cazadores-recolectores. La observación de condiciones ecológicas variables

\footnotetext{
7 Los vuelos se hicieron por acuerdo con el Club Aéreo de Calama a través de la cortesía del Sr. Roberto Harvey, Chuquicamata. Especialmente agradezco a los pilotos, Dr. Juan Rojas y Sr. Juan Mediano, por su experiencia y cooperación.
} 
en la localidad sugirió que en ciertos tiempos esta área de recursos era de fácil acceso a las poblaciones que vivían a lo largo del río Loa.

Desde el aire, uno se sorprende ante el número de pequeños arroyos que desaguan en las vegas del río Loa desde la vertiente noroccidental de los cerros de Tuina. Hubo una cantidad de agua considerable en un pequeño salar a lo largo de uno de estos arroyos, y aparentemente la vegetación seca, que previamente se creyó que existía sólo en las partes altas de los cerros, se extendía vertiente abajo hasta unos $20 \mathrm{~km}$ de las vegas del río Loa. Una prospección hecha en jeep confirmó esta sospecha.

La actual vegetación de las vertientes de los cerros de Tuina es en su mayoría xerófita, y consiste en rica-rica y otras especies no comestibles por el hombre. Esta área se considera por lo tanto, un recurso potencial de pasto más que un recurso para la alimentación humana, y nuestro proyecto de caza confirmó esta suposición.

Localizamos 13 guanacos en un área de por lo menos $25 \mathrm{~km}^{2}$ en el lado sudoriental de los cerros de Tuina, a unos $50 \mathrm{~km}$ al sureste del río Loa. Los guanacos comen las hojas verdes de la vegetación xerófila, buscando este forraje en cárcavas cuando la vegetación de los interfluvios está seca. También comen paja $i c h u$, pero en la actualidad ésta se restringe sólo a las partes más altas dentro del rango de altura en donde crece, es decir a unos 11.000 pies. Basándonos en nuestras observaciones y comunicaciones de informantes, estimamos que en la actualidad viven unos 30 a 40 guanacos en 200 a $300 \mathrm{~km}^{2}$, área que abarca los cerros de Tuina entre el Llano de la Paciencia por el sureste y el camino de Tuina por el noroeste. La cantidad de guanacos que el área puede sostener es mayor que la cantidad que sostiene actualmente en una proporción de 1:3, puesto que por lo menos hay unas 150 llamas, ovejas, cabras y unos pocos burros salvajes, que están aumentando el número actual de animales. De este modo, las actuales manadas locales de guanacos son probablemente remanentes de manadas precolombinas más grandes.

El cambio climático también ha restringido la disponibilidad del área y por lo tanto varió el número de guanacos en años recientes. William E. Rudolph (com. pers.), informa que hacen unos 50 años atrás habían guanacos y vertientes en áreas ahora totalmente estériles. El autor descubrió huellas de camélidos en áreas alejadas de vegetación y de rutas de comercio. Se obtuvo información adicional sobre las relaciones entre clima, vegetación y fauna por medio de la observación de los efectos causados por precipitación copiosa en 1970-1971.

Llovió sobre el área de asentamientos de la vega del Loa tres veces durante la temporada de campo de 17 meses, y aún más a menudo en las tierras altas. Esto es extremadamente raro en la región, ya que generalmente, se lee que la precipitación anual promedio en Calama es menos de $1 \mathrm{~mm}$. Cada lluvia en Calama estaba acompañada de nieve en las tierras altas. La precipitación del 11 de junio de 1971 fue particularmente interesante porque llovió en Calama y nevó durante dos horas en Chuquicamata, por primera vez en 12 años. La nieve se encontraba al día siguiente a una altura relativamente baja de 9500 pies en el camino a Ojos de San Pedro, sugiriendo que esta área estaba cubierta por unas pocas pulgadas de nieve. El 13 de junio encontramos una capa casi continua de nieve de unas dos pulgadas, en las partes más altas de las vertientes sudorientales de los cerros de Tuina a una altura aproximada de 10000 pies. La nieve se mantuvo cerca de una semana en estas áreas bajas y en las partes más altas de la cordillera unas cuatro semanas.

El 17 de junio, experimentamos caídas de nieve a unos 7800 pies en el área del río Loa y al día siguiente fotografiamos nieve en la zona de guanacos a unos 10800 pies de altura.

En mayo de 1970 ocurrieron precipitaciones similares, aunque menos intensas, en la vega del Loa y en los cerros de Tuina. El invierno boliviano, cuando cae precipitación de verano en forma de nieve en las tierras altas en los meses de enero y febrero, pareció ser relativamente intenso.

El reciente período de aumento de precipitación abarcó las tierras altas de los Andes, los cerros de Tuina y sus vertientes que enfrentan el Loa, y a veces, el área de vegas del Loa. La precipitación nunca fue torrencial y nunca causó pantanos. Siempre estuvo acompañada por períodos relativamente largos de bajas temperaturas y gran nubosidad con estratos y estratocúmulos. El aumento del agua de subsuelo y vertientes se notó en los cerros de Tuina a través del aumento de vegetación. Aparentemente, la lluvia del 11 de junio volvió verde la vegetación seca de las partes bajas del área de guanacos, en las vertientes noroccidentales de los cerros de Tuina. Una semana 
después de la lluvia, apareció la vegetación verde y permaneció así hasta mediados de agosto, cuando terminó la temporada de campo.

La nieve en el área de guanacos, indudablemente motivó a los animales a migrar hacia tierras más bajas y secas, y probablemente forzó a la vicuña a salir de la alta cordillera. Este tipo de migración de fauna ha ocurrido otras veces, de acuerdo a información de mineros de la región.

Creemos que los cambios meteorológicos recién descritos muestran un ejemplo de cambio ambiental bajo condiciones pluviales y subpluviales. Los fenómenos meteorológicos ocurridos en el último período de campo tuvieron su paralelo en la península de Santa Elena, con cambios aún más violentos (Sarma y Cole, com. pers.) y así ambas áreas parecen estar actuando de acuerdo a un patrón frío pluvial panandino (Sarma, com. pers.). Por lo tanto, nos es permitido utilizar el tipo de clima descrito y sus efectos como modelo de condiciones pluviales y subpluviales durante parte del Período Chiu Chiu. Este modelo climático, combinado con hipótesis y observaciones sobre hábitos de fauna, economía de subsistencia, patrón de asentamiento y otros factores ya mencionados, pueden resumirse en la siguiente serie de hipótesis:

1) Durante períodos secos, como el actual, la población de Chiu Chiu explotó una amplia área dentro de un patrón de trashumancia estacional que incluía permanencias estacionales en el río Loa donde estuvieran presentes recursos de materia prima lítica, agua dulce, madera para hacer fuego, piedras para construcción, pastos, rizomas de juncos, fauna pequeña como roedores y aves acuáticas. Otras permanencias estacionales incluían Ojos de San Pedro, rica en fauna pero quizás demasiado fría en invierno como para que la gente se quedara por temporadas completas. Debieron existir suficientes guanacos y vizcachas en las partes altas de los cerros de Tuina, que justificarían algunos períodos de asentamientos allí. La ausencia de buena materia prima lítica, sin embargo, tendería a restringir el tiempo de permanencia en esta área. La escasez de agua, especialmente después de períodos prolongados de sequía, también pudo constituir un problema. La falta de vertientes en los cerros de Tuina forzaría a la gente a descender al área de Hierba Buena o al río Loa en busca de agua dulce. Esto último permitiría además, completar su equipo de herramientas líticas con nuevos artefactos obtenidos en las canteras del salar de Talabre.
2) El factor tiempo asociado a fenómenos de agua subterránea es complejo, y hay información suficiente para pensar que el tipo de escurrimiento era tan lento que las vertientes del pie de los cerros podrían haber estado activas, mientras el área del río Loa estaba extremadamente seca. En ese caso, la población pudo haber permanecido en los cerros de Tuina quizás por varios años consecutivos, mandando grupos hacia las canteras del Loa cuando fuera necesario. Esto podría reflejarse en la gran cantidad de sitios Chiu Chiu de canteras-talleres en el área y en los vacíos dentro de la seriación de la secuencia de la fase Chiu Chiu.

3) Claramente, durante los períodos secos la vega del Loa y los cerros de Tuina fueron dos áreas distintas de recursos, separadas por lo menos por $20 \mathrm{~km}$ de extremo desierto.

4) Durante los períodos pluviales, las zonas de Tuina y el Loa probablemente formaron un área única y continua de recursos. De acuerdo a nuestro modelo, un período pluvial estaba acompañado por temperaturas más bajas, aumento de nubosidad, aumento absoluto de precipitación, que durante máximos pluviales podría caer en el área del Loa, y una disminución en la evaporación, como resultado del aumento de precipitación. El área del Loa así como la de Tuina tendrían, por lo tanto, un aumento en forma geométrica en la densidad vegetacional como resultado de la germinación de semillas latentes, y en ambas áreas pudo existir colonización de plantas. Podríamos esperar que la distribución de paja $i c h u$ se haya extendido hacia menores alturas, y el Solanum, ahora encontrado en áreas más altas, húmedas y frías, pudo también migrar hacia tierras más bajas, quizás invadiendo el área del Loa. Las manadas de guanacos habrían aumentado de tamaño en los cerros de Tuina y, lo que es más importante, habrían llegado a tierras más bajas, siguiendo la vegetación que se extendía cercana al río Loa mientras persistían las condiciones pluviales. La caída de nieve en las tierras altas también habría forzado a los guanacos a bajar. Un pluvial alto pudo extender la vegetación de los cerros hacia el río. Esto es posible, puesto que implica un traslado de sólo $500 \mathrm{~m}$ verticales y $20 \mathrm{~km}$ laterales, lo que pudo ser facilitado por el aumento de agua subterránea. De este modo las dos áreas de recursos se debieron juntar, puesto que los recursos habrían aumentado y estarían ampliamente dispersos, reduciendo el número y espacio de microambientes explotados. Por ejemplo, uno podría vivir en el extremo norte del área del Loa y cazar 
guanacos cerca de Angostura, o quizás más cerca al lugar del asentamiento. Esto evitaría la necesidad de abandonar el área periódicamente, permitiendo una mayor permanencia en el asentamiento. Tal aumento de sedentarismo podría estar reflejado por la profundidad de las basuras en sitios como RAnL-140. Los aumentos de población podrían estar reflejados en sitios más grandes como RAnL-217.
Más que aventurarse en un determinismo climático, el modelo en curso está basado en observaciones de campo, referencias de laboratorio y consideraciones teóricas. El modelo puesto a prueba con información adicional será revisado y descartado si fuera necesario. Hasta ahora, sin embargo, parece ser la mejor forma de manejar los datos.

\section{REFERENCIAS CITADAS}

BARFIELD, L., 1961. Recent discoveries in the Atacama Desert and the Bolivian altiplano. American Antiquity 27 (1): 93-100.

BIRD, J., 1943. Excavations in Northern Chile. Anthropological Papers of the American Museum of Natural History, vol. 38, part 4.

LANNING, E., 1967. Peru before the incas. Englewood Cliffs, Prentice-Hall.
LANNING, E. y E. HAMMEL, 1961. Early lithic industries of western South America. American Antiquity 27 (2): 139-154.

SARMA, A., 1969. Cultural implications of Upper Pleistocene and Holocene ecology of the Santa Elena península, Ecuador. $\mathrm{Ph}$. D. dissertation. Department of Anthropology, Columbia University, Nueva York. 